\title{
Game Based Energy Cost Optimization for Unmanned Aerial Vehicle Communication Networks
}

\author{
Changhua Yao $\mathbb{D}$, Lei Zhu $(\mathbb{D}$, Lei Wang, and Junye Meng \\ The Army Engineering University of PLA, Nanjing, China \\ Correspondence should be addressed to Lei Zhu; LeiZhuxueshuj@163.com
}

Received 26 November 2017; Revised 29 January 2018; Accepted 26 February 2018; Published 10 April 2018

Academic Editor: Eduardo Rodriguez-Tello

Copyright (C) 2018 Changhua Yao et al. This is an open access article distributed under the Creative Commons Attribution License, which permits unrestricted use, distribution, and reproduction in any medium, provided the original work is properly cited.

\begin{abstract}
Due to the limited transmission power, the data transmission between the unmanned aerial vehicle and the ground station often needs the synergetic forwarding. The optimization of the synergetic forwarding organization is important to the performance of the unmanned aerial vehicle communication networks. This paper aims to optimize the energy cost using the synergetic forwarding mode in the unmanned aerial vehicle communication networks. To reduce the expensive information exchange and improve the robust of the network, we put forward an energy cost orient forwarding allocation approach using game based intelligent algorithm. The theoretic analysis and simulation results verify that the put forward method could achieve optimal energy cost communication organization.
\end{abstract}

\section{Introduction}

The unmanned aerial vehicle (UAV) is the hot research point recently [1]. With its flexibility and low cost, the UAV could complete many kinds of work which are hard to the human, such as dangerous detection, long-time monitoring, and remote rescuing. Limited by single UAV's ability, the UAV swarm consisting of multiple UAVs draws more and more attention [2]. Besides many key issues, the communication organization for the UAVs is a basic problem.

There are some researches on the UAV communication networks. In [3], Rosati et al. proposed a speed-aware routing algorithm that is applied in the context of high-speed UAVs. In [4], Zhu et al. studied the design and evaluation of airborne communication networks. In [5], Ortiz et al. studied the design and development of a robust ATP subsystem for the altair UAV-to-ground lasercomm $2.5 \mathrm{Gbps}$ demonstration. Luo et al. proposed a distributed gateway selection algorithm for UAV networks in [6]. Yin et al. put forward queuing models for deciding the optimal choice of UAVs to forward packets in [7]. In [8], Saleem et al. stated the integration of cognitive radio technology with unmanned aerial vehicles, including the important issues and research challenges. In [9], Choi et al. paid attention to the energy-efficient maneuvering and communication of a single UAV-based relay. Author Puri made a survey of unmanned aerial vehicles (UAV) for traffic surveillance in [10]. In [11], Bekmezci et al. made a survey on the flying ad hoc networks. In [12], Wang et al. studied the position unmanned aerial vehicles in the mobile ad hoc network. In [13], Ono et al. studied the relay network based on unmanned aircraft network.

With the ground station system supported [5], the UAV communication network could solve the fast information transmission problem well. However, common UAV usually would be limited by energy, the long-distance data transmission is not a good way since the pow cost increases with the distance fast. In addition, the data transmission would not be done when the UAV is out of the range. As a result, the synergetic forwarding in the UAV communication network is necessary to pay attention to [14]. Another important issue in the UAV communication network is the pow cost, which is very sensitive to the UAV, especially for the ones supported by the battery. How to optimize the synergetic forwarding communication organization considering the energy cost is important to the whole system. In [9], Choi et al. studied the energy-efficient maneuvering and communication of a single UAV-based relay in depth. In [14], Wu et al. made important research on the movement design, by considering the energy cost. A mobile forwarding approach was proposed for the monitored data transmission. However, this work focused 
on a single base station's movement optimization; it is not a multiuser network. In [15], a power consumption optimal synergetic forwarding scheme was put forward to improve the system's lifetime by Li et al.; nevertheless, the research in [14] mainly focuses on single UAV's movement design, not for the UAV communication network. The approach proposed in [15] is a centralized one, not the focused point in our work. Due to the UAV's high dynamism, the centralized optimization approach would not be capable of dealing with the topology change and information exchange problems. To the best of our knowledge, the energy cost improvement issue in the UAV communication network has not been well solved in existing works.

In this paper, we aim to study the energy cost improvement issue in the UAV communication network. We put forward an energy cost orient forwarding allocation approach to achieve the optimal solution to the UAV communication networks energy optimization issue. The theoretic analysis is presented by modeling this problem as a game [16]. The experiments are carried out to verify the theoretic result and the performance of proposed intelligent learning algorithm.

\section{Network Model and Problem Formulation}

A scenario where UAV communication networks are supported by the ground communication station system [5] is shown in Figure 1. The UAV communication networks consist of $M$ common UAVs (CUAVs) and $N$ forwarding UAVs (FUAVs). Usually, the CUAVs' communication would be limited by their energy; it is hard for CUAVs to communicate with the grand stations directly. The FUAVs are usually the ones supported by fuel, which have the ability to communicate with the ground stations directly. In the UAV communication networks, the FUAVs would work as the forwarding node for the CUAVs. With different ground stations and different FUAVs' communication devices, the link bandwidths would also be different. As shown in the scenario in Figure 1, the bandwidths of FUAVs might be $6 \mathrm{MHz}, 15 \mathrm{MHz}, 20 \mathrm{MHz}$, and so on.

Note that the topology of the UAV communication network is varying all the time. As shown in Figure 1, the CUAVs, FUAVs, and the mobile ground stations are all moving. The dynamic topology is the character of the UAV communication networks, and the distances between CUAVs and FUAVs vary. As a result, the transmission powers cost for the communication would also change to meet the required data transmission quality. The selection of FUAV to forward the data would be critical for the CUAVs' energy cost. The following attributes have important effect on the energy cost: the distance to FUAV, the transmission channel quality, and the bandwidth. As shown in Figure 1, CUAV1 could select one of the four FUAVs as the communication forwarding node to the ground station system. The selection would be determined by the expected energy cost. Define $\Pi_{F}=\{1,2, \ldots, N\}$ and $\Pi_{C}=\{1,2, \ldots, M\}$ as the set of FUAVs and CUAVs. Define $B_{F}=\left\{B_{1}, B_{2}, \ldots, B_{N}\right\}$ as the bandwidths vector. The bandwidth allocation between CUAVs could be designed by different scheme; it is assumed that the bandwidth would be equally allocated by the connected CUAVs in this paper for the simplification. The CUAVs' selection of forwarding node would be decided by the CUAVs' traffic requirement and the distance to the FUAVs.

Based on the Shannon theory, assuming that $C_{m}$ selects $F_{n}$ as the forwarding node, the achieved data transmission rate would be

$$
R_{m, n}=B_{m, n} \log \left(1+\frac{E_{m, n} \delta_{m, n}{ }^{-\gamma_{m, n}} \rho_{m, n}}{B_{m, n} N_{0}}\right),
$$

where $N_{0}$ is the noise power spectrum density, $\delta_{m, n}$ is the distance between $C_{m}$ and $F_{n}, \gamma_{m, n}$ is the path-loss exponent between $C_{m}$ and $F_{n}$, and $\rho_{m, n}$ is the instantaneous random component of the path loss. Then, the energy cost (EC) of $C_{m}$ would be

$$
E_{m, n}=\frac{B_{n} N_{0}\left(\exp ^{\left(\left|\Omega_{n}\right| R_{m, n} / B_{n}\right)}-1\right)}{\left|\Omega_{n}\right| \delta_{m, n}^{-\gamma_{m, n}} \rho_{m, n}},
$$

where $\left|\Omega_{n}\right|$ is the number of CUAVs selected $F_{n}$ as the forwarding node.

It should be noted that, in the UAV communication networks, the best forwarding node selection would be not only affected by some CUAV itself, but also determined by other UAVs' selection. With the whole UAV communication networks, the goal is that the sum of the energy cost is minimized:

$$
\text { Problem: } \min \quad E_{\text {net }}=\sum_{m=1}^{M} E_{m},
$$

that is,

$$
\text { Problem: } \max \quad-E_{\text {net }}=-\sum_{m=1}^{M} E_{m} .
$$

The main challenges of this problem are as follows: first, the optimization for the forwarding node allocation in UAV communication networks is the combinational optimization issue. The searching approach could achieve the best combination, but the computing complex would increase fast when the UAV network increases. The possible combination would be $7^{35}=3.79 \times 10^{29}$ in relatively small 35 common UAV and 7 forwarding UAV communication networks. With the genetic algorithm, ant colony algorithm, and the like, the performance of the optimization could not be guaranteed. Second, the information exchange required by the centralized optimization approach would not be practical, for the limited communication capability and the limited time. Third, the dynamism of the UAV communication network brings serious problem to the centralized optimization, including the dynamism of topology and the dynamism of environment. In the following sections, we solve this problem based on the game theory, which could achieve the optimal state of the network without the centralized optimization.

\section{The Energy Cost Orient Forwarding Allocation Approach}

In this section, we put forward an energy cost orient forwarding allocation approach (ECOFAA) to optimize the 


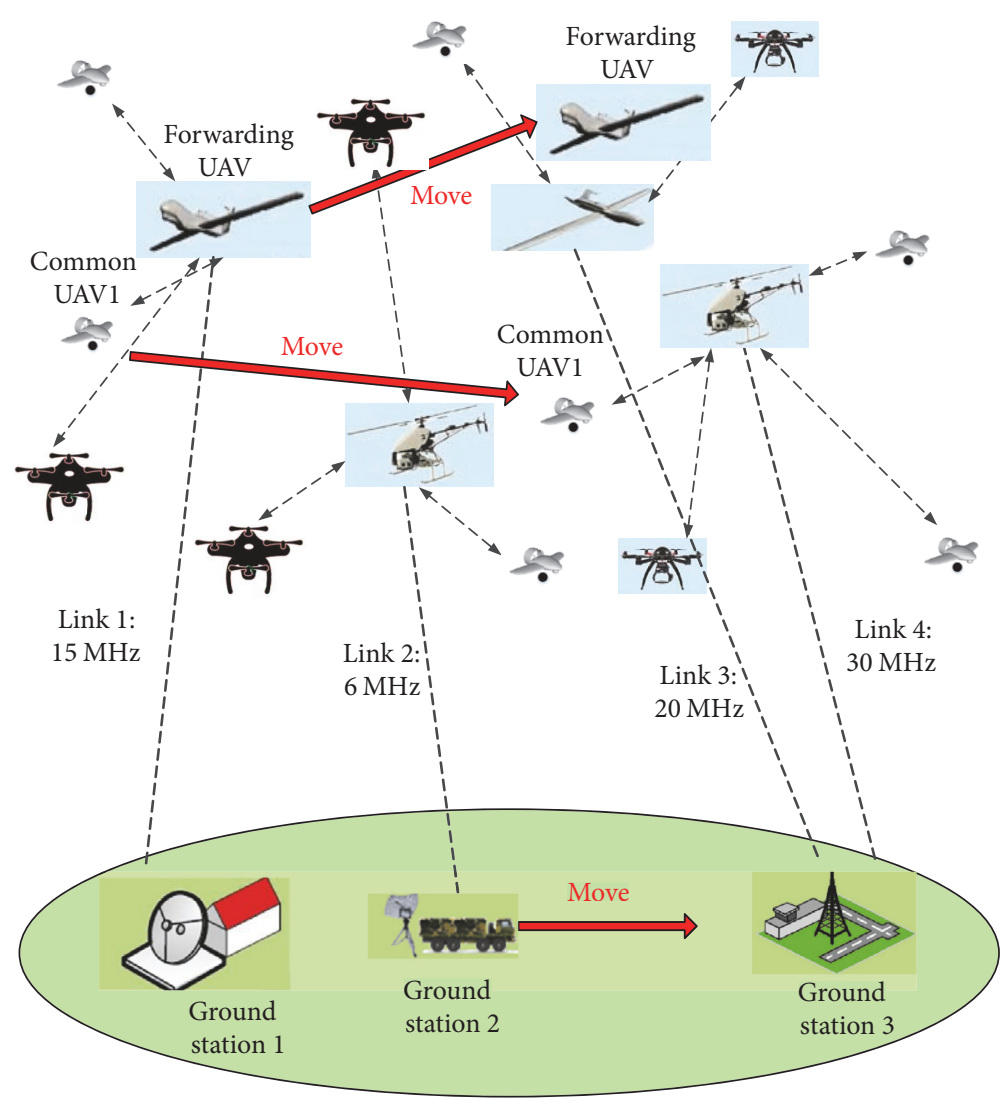

FIGURE 1: Ground system supported UAV communication network.

energy cost optimization of UAV communication network. The allocation approach is shown in Figure 2.

In the approach in Figure 2, the parameter $\beta>0$ plays the role of adjusting with the change of environment. Note that the probabilistic selection scheme [17] is adopted to avoid the suboptimal trap problem in best-response algorithm [18] and the like.

Remark 1. The put forward energy cost orient forwarding allocation approach is a distributed method rather than a centralized one. All the UAVs make their action decision by themselves rather than by some control center. This is important to the practicability of the approach in the dynamic environment that the UAV communication network faces.

Theorem 2. The put forward energy cost orient forwarding allocation approach would achieve the minimal energy cost and stable network state.

Proof. With the UAV communication network shown in Figure 1, when the UAVs adopt the action updating strategies as the put forward approach, the system could be seen as a game model as follows, and each CUAV could be seen as a player in the game. Define the energy cost orient forwarding allocation (ECOFA) game as follows:

$$
G=\left\{\Pi_{C}, \Pi_{F}, \Psi, A\right\},
$$

where $\Psi$ is the topology relationship of the UAV communication networks, among which $N_{m, n} \subset \Psi$ is the communication distance between $F_{n}$ and $C_{m} . C_{m}$ could communicate with $F_{n}$ if $N_{m, n}=1$; otherwise, $N_{m, n}=0 . A=A_{1} \otimes A_{2} \otimes$ $\cdots \otimes A_{M}$ is the action profiles of all the nodes, where $\otimes$ is the Cartesian product and $A_{m}$ is the possible actions of $C_{m}$. Define $C_{m}$ 's action as $a_{m} \in A_{m} . u_{m}$ is the utility function of $C_{m} \cdot u_{m}\left(a_{m}, a_{-m}\right)$ would be $C_{m}$ 's utility when $C_{m}$ 's action is $a_{m}$ and other players' action is $a_{-m}$. Nei $i_{m}$ is the set of CUAVs:

$$
N e i_{m}=\left\{i \in \Pi_{C}: \text { if } N_{m, j}=1, N_{i, j}=1, \forall j \in \Pi_{F}\right\} .
$$

In the put forward ECOFA game, inspired by the synergy design in networks [18-20], $C_{m}$ 's utility function would be as follows:

$$
u_{m}\left(a_{m}, a_{-m}\right)=-E_{m}-\sum_{i \in N e i_{m}} E_{i}
$$

According to potential game theory [19], define the potential function of the ECOFA game as follows:

$$
\begin{aligned}
\Gamma\left(a_{m}, a_{-m}\right) & =-E_{\text {net }}=-\sum_{m=1}^{M} E_{m} \\
& =-E_{m}-\sum_{i \in N e i_{m}} E_{i}-\sum_{i \in\left\{\Pi_{C} \backslash N e i_{m}\right\}} E_{i}
\end{aligned}
$$




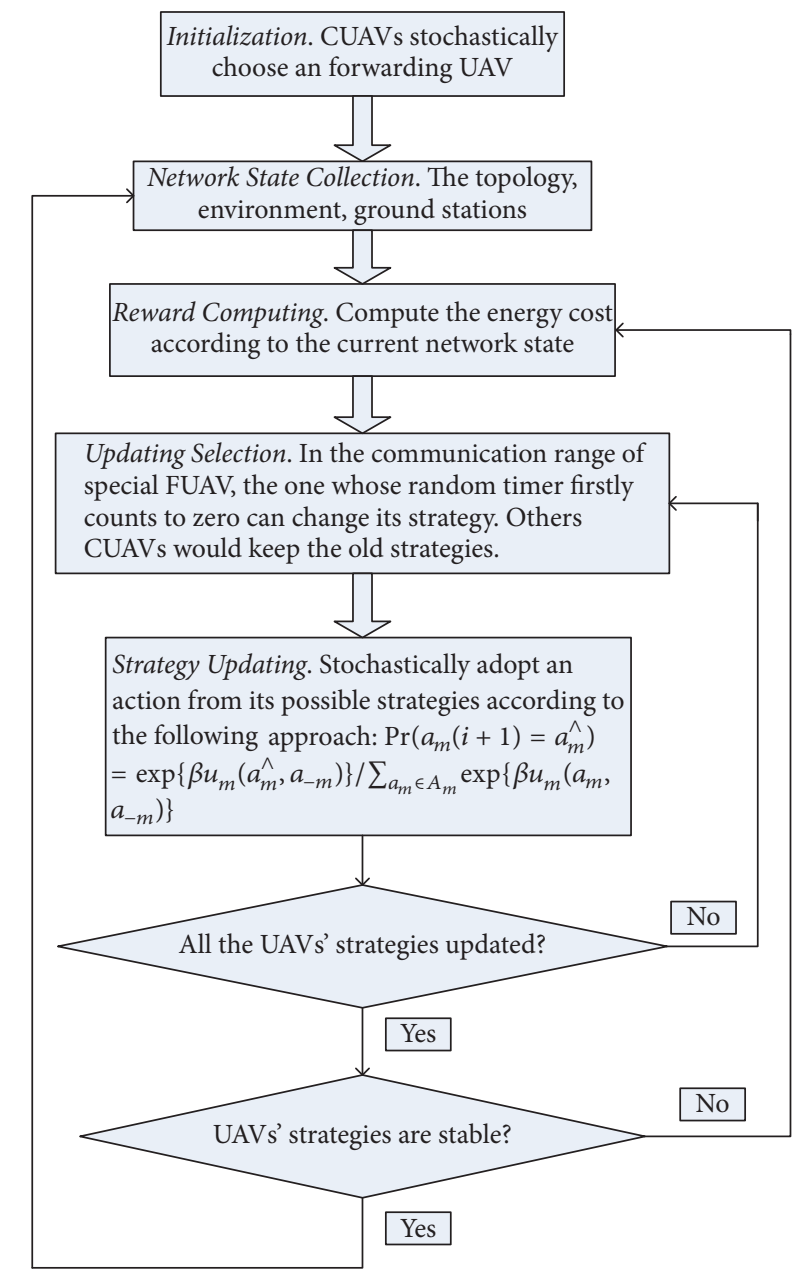

FIGURE 2: The energy cost orient forwarding allocation approach.

Assume that $C_{m}$ updates its action from $a_{m}$ to $a_{m}^{\wedge}$ and other UAVs hold their actions; based on the definition of $N e i_{m}$, the change of the potential function would be computed as follows:

$$
\begin{aligned}
\Delta \Gamma= & \Gamma\left(a_{m}, a_{-m}\right)-\Gamma\left(a_{m}^{\wedge}, a_{-m}\right)=-E_{\text {net }}+E_{\text {net }}^{\wedge} \\
= & -E_{m}-\sum_{i \in N e i_{m}} E_{i}-\sum_{i \in\left\{S_{C} \backslash N e i_{m}\right\}} E_{i} \\
& -\left(-E_{m}^{\wedge}-\sum_{i \in N e i_{m}} E_{i}^{\wedge}-\sum_{i \in\left\{S_{C} \backslash N e i_{m}\right\}} E_{i}^{\wedge}\right) \\
= & E_{m}^{\wedge}-E_{m}+\sum_{i \in N e i_{m}} E_{i}^{\wedge}-\sum_{i \in N e i_{m}} E_{i}+\sum_{i \in\left\{\Pi_{C} \backslash N e i_{m}\right\}} E_{i}^{\wedge} \\
& -\sum_{i \in\left\{\Pi_{C} \backslash N e i_{m}\right\}} E_{i}=E_{m}^{\wedge}-E_{m}+\sum_{i \in N e i_{m}} E_{i}^{\wedge}-\sum_{i \in N e i_{m}} E_{i} \\
= & u_{m}\left(a_{m}, a_{-m}\right)-u_{m}\left(a_{m}^{\prime}, a_{-m}\right)=\Delta u .
\end{aligned}
$$

According to analysis [19], the put forward ECOFA game is an exact potential game. Then, it has at least one pure strategy NE point, and the optimal state of potential function in the ECOFA game would be a Nash equilibrium point. According to the design of the potential function, the optimal energy cost network state would also be a Nash equilibrium point of the ECOFA game. With the network state transmission in the put forward approach, suppose $a_{m}=$ $F_{m}(i)$ as the $C_{m}$ 's action in the $i$ th iteration in the put forward approach. Define $\Omega(i)=\left(F_{1}(i), F_{2}(i), \ldots, F_{m}(i), \ldots, F_{M}(i)\right)$ as the network state, which is a discrete time Markov process with a unique stationary distribution [20]. Define the unique stationary distribution of CUAVs' strategy profile as $\overline{\mathbf{a}}=$ $\left\{\overline{a_{1}}, \overline{a_{2}}, \ldots, \overline{a_{M}}\right\}$, which would be given by the following:

$$
\pi(\overline{\mathbf{a}})=\frac{\exp \{\beta \Gamma(\overline{\mathbf{a}})\}}{\sum_{\mathbf{a} \in A} \exp \{\beta \Gamma(\mathbf{a})\}},
$$

where $\Gamma(\mathbf{a})$ is the potential function of the game. $A=A_{1} \otimes$ $A_{2} \otimes \cdots \otimes A_{M}$ is the set of strategies of all the UAVs.

Define $\Omega(i+1)=\mathbf{a}_{2}, \Omega(i)=\mathbf{a}_{1}$. Define the transition probability from state $\mathbf{a}_{1}$ to $\mathbf{a}_{2}$ as $P_{\mathbf{a}_{1}, \mathbf{a}_{2}}$, the transition probability from state $\mathbf{a}_{2}$ to $\mathbf{a}_{1}$ as $P_{\mathbf{a}_{2}, \mathbf{a}_{1}}$. Supposing a CUAV updating the FUAV chosen from $a_{m}(i)=F_{m}(i)$ to $a_{m}^{\wedge}(i+$ $1)=F_{m}^{\wedge}(i+1)$, then the UAV communication network 
state would be changed from $\mathbf{a}_{1}$ to $\mathbf{a}_{2}$, that is, from $\Omega(i)=$ $\left(F_{1}(i), F_{2}(i), \ldots, F_{m}(i), \ldots, F_{M}(i)\right)$ to $\Omega(i+1)=\left(F_{1}(i+1), F_{2}(i+\right.$ $\left.1), \ldots, F_{m}^{\wedge}(i+1), \ldots, F_{M}(i+1)\right)$.

With the UAV communication network consisting of $M$ CUAVs, the probability of $C_{m}$ updating its forwarding FUAV would be $1 / M$. Then,

$$
\begin{aligned}
\pi & \left(\mathbf{a}_{1}\right) P_{\mathbf{a}_{1}, \mathbf{a}_{2}} \\
= & {\left[\frac{\exp \left\{\beta \Gamma\left(\mathbf{a}_{1}\right)\right\}}{\sum_{\mathbf{a} \in A} \exp \{\beta \Gamma(\mathbf{a})\}}\right] } \\
& \times\left[\left(\frac{1}{M}\right)\left(\frac{\exp \left\{\beta u_{m}^{\wedge}\left(a_{m}^{\wedge}, a_{-m}\right)\right\}}{\sum_{a_{m} \in A_{m}} \exp \left\{\beta u_{m}\left(a_{m}, a_{-m}\right)\right\}}\right)\right] \\
= & \frac{\exp \left\{\beta\left(\Gamma\left(\mathbf{a}_{1}\right)+u_{m}^{\wedge}\left(a_{m}^{\wedge}, a_{-m}\right)\right)\right\}}{M \times \sum_{\mathbf{a} \in A} \exp \{\beta \Gamma(\mathbf{a})\} \times \sum_{a_{m} \in A_{m}} \exp \left\{\beta u_{m}\left(a_{m}, a_{-m}\right)\right\}} . \\
\pi & \operatorname{Similarly,} \\
= & {\left[\frac{\exp \left\{\beta \Gamma\left(\mathbf{a}_{2}\right)\right\}}{\sum_{\mathbf{a} \in A} \exp \{\beta \Gamma(\mathbf{a})\}}\right] } \\
& \times\left[\left(\frac{1}{M}\right)\left(\frac{\exp \left\{\beta u_{m}\left(a_{m}, a_{-m}\right)\right\}}{\sum_{a_{m} \in A_{m}} \exp \left\{\beta u_{m}\left(a_{m}, a_{-m}\right)\right\}}\right)\right] \\
= & \frac{\exp \left\{\beta\left(\Gamma\left(\mathbf{a}_{2}\right)+u_{m}\left(a_{m}, a_{-m}\right)\right)\right\}}{M \times \sum_{\mathbf{a} \in A} \exp \{\beta \Gamma(\mathbf{a})\} \times \sum_{a_{m} \in A_{m}} \exp \left\{\beta u_{m}\left(a_{m}, a_{-m}\right)\right\}} .
\end{aligned}
$$

According to the character of the exactly potential game, we have

$$
\Gamma\left(\mathbf{a}_{1}\right)-\Gamma\left(\mathbf{a}_{2}\right)=u_{m}\left(a_{m}, a_{-m}\right)-u_{m}^{\wedge}\left(a_{m}^{\wedge}, a_{-m}\right) .
$$

Then we have

$$
\begin{aligned}
& \exp \left\{\beta\left(\Gamma\left(\mathbf{a}_{1}\right)+u_{m}^{\wedge}\left(a_{m}^{\wedge}, a_{-m}\right)\right)\right\} \\
& =\exp \left\{\beta\left(\Gamma\left(\mathbf{a}_{2}\right)+u_{m}\left(a_{m}, a_{-m}\right)\right)\right\} .
\end{aligned}
$$

Thus,

$$
\pi\left(\mathbf{a}_{1}\right) P_{\mathbf{a}_{1}, \mathbf{a}_{2}}=\pi\left(\mathbf{a}_{2}\right) P_{\mathbf{a}_{2}, \mathbf{a}_{1}} .
$$

As a result,

$$
\begin{aligned}
\sum_{\mathbf{a}_{1} \in A} \pi\left(\mathbf{a}_{1}\right) P_{\mathbf{a}_{1}, \mathbf{a}_{2}} & =\sum_{\mathbf{a}_{1} \in A} \pi\left(\mathbf{a}_{2}\right) P_{\mathbf{a}_{2}, \mathbf{a}_{1}}=\pi\left(\mathbf{a}_{2}\right) \sum_{\mathbf{a}_{1} \in A} P_{\mathbf{a}_{2}, \mathbf{a}_{1}} \\
& =\pi\left(\mathbf{a}_{2}\right) .
\end{aligned}
$$

Based on the analysis in [20], the put forward approach has the stationary distribution. Define that $\mathbf{a}^{\#}$ is the CSUVs' forwarding choosing selection in the optimal energy cost network state; then

$$
\mathbf{a}^{\#}=\underset{\mathbf{a} \in A}{\arg \min } E_{\text {net }}=\underset{\mathbf{a} \in A}{\arg \min } \Gamma(\mathbf{a}) .
$$

According to the analysis above, the put forward approach would converge to a unique stationary distribution
TABLE 1: The simulation parameters.

\begin{tabular}{lc}
\hline Number of CUAVs & 25 \\
Number of FUAVs & 6 \\
The communication data rate & {$[6,10,15,20,25,32] \mathrm{MHz}$} \\
The bandwidths of FUAVs & $1 \mathrm{Mbit} / \mathrm{s}$ \\
The noise power & $-130 \mathrm{~dB}$ \\
The path-loss exponent & 2 \\
\hline
\end{tabular}

$\pi(\overline{\mathbf{a}})=\exp \{\beta \Gamma(\overline{\mathbf{a}})\} / \sum_{\mathbf{a} \in A} \exp \{\beta \Gamma(\mathbf{a})\}$. When $\beta \rightarrow \infty$, $\exp \left\{\beta \Gamma\left(\mathbf{a}^{\#}\right)\right\} \gg \exp \{\beta \Gamma(\mathbf{a})\}, \forall \mathbf{a} \in\left\{A \backslash \mathbf{a}^{\#}\right\}$.

The probability of achieving best energy cost network state $\mathbf{a}^{\#}$ will be

$$
\lim _{\beta \rightarrow \infty} \pi\left(\mathbf{a}^{\#}\right)=\frac{\exp \left\{\beta \Gamma\left(\mathbf{a}^{\#}\right)\right\}}{\sum_{\mathbf{a} \in A} \exp \{\beta \Gamma(\mathbf{a})\}}=1 .
$$

The above result shows that the put forward intelligent learning approach would converge to the optimal energy cost state of the UAV communication network. In addition, the state would be stable since it is a Nash equilibrium point where none of the players would like to change its strategy. Hence, the theorem is proved.

The above analysis proves that the put forward approach would converge to the optimal network state. Importantly, the proposed approach is an online method which could adjust the UAVs' strategies according to the change of the environment, the change of the topology, and so on. In all, the proposed approach is a distributed and online optimization method which is suitable to the dynamic UAV communication network.

\section{Numeric Results and Discussion}

To verify the performance of put forward energy cost orient forwarding allocation approach (ECOFAA), the comparison between the ECOFAA and some existing algorithms have been carried out. The simulation is done by Matlab. The simulation parameters are depicted in Table 1 and Figure 3.

The parameter setting in the simulation is not specialized. The parameters such as number of CUAVs, number of FUAVs, the communication data rate, the bandwidths of FUAVs, the noise power, and the path-loss exponent could all be changed. The parameter setting is not sensitive to the proposed approach.

The simulation results on the energy cost have been shown in Figure 4. To show the details of the course in the put forward ECOFAA approach, the energy cost of three randomly chosen CUAV s are observed. As shown in Figure 4, all of the three CUAVs' energy costs converge to a stable value at last, which proves that the CUAVs' forwarding selection actions would not vary again after the proposed ECOFAA converges. It should be noted that other CUAVs forwarding selection could directly or indirectly affect some CUAV's EC in the UAV communication network, so the energy cost of the observed CUAVs would not be stable during the updating. To verify the proposed approach in an average aspect, 1500 


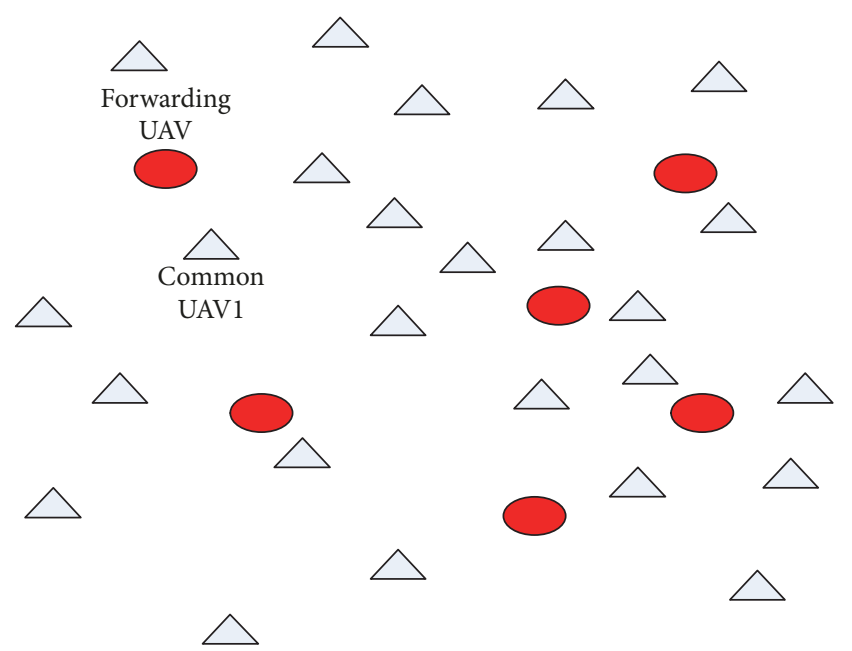

FIGURE 3: The topology of the simulation network consists of 6 FUAVs and 25 CUAVs.

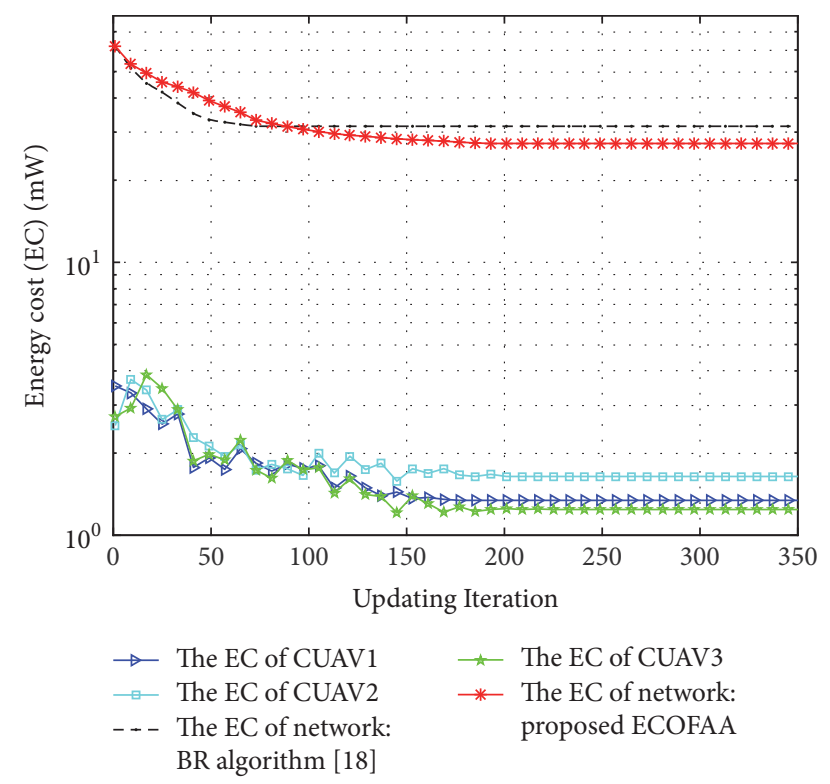

FIgURE 4: The energy cost of CUAVs in the updating procedure of proposed ECOFAA.

independent simulating experiments have been carried out, and the average numeric result has been shown. It could be seen that the put forward ECOFAA outperformed the best-response learning algorithm [18] when the learning converges. The proposed ECOFAA achieves lower energy cost. The best-response learning algorithm would converge faster than the proposed ECOFAA, but the total energy cost would be higher. That means the best-response learning algorithm could not achieve the best FUAV forwarding allocation state for the UAV communication network. At the beginning, the forwarding UAVs are randomly allocated, and the total energy cost of the whole UAV communication network would be relatively high. After the forwarding UAV selection updating by the proposed ECOFAA, the total energy cost of the network would be reduced obviously. Importantly, the total energy cost would not vary after the proposed method converges. The simulation result of energy cost converging verifies that proposed FUAV allocation approach would be stable.

\section{Conclusion}

In this paper, we studied on the UAV communication networks energy optimization issue, which is critical to the whole UAV network. We put forward an energy cost orient forwarding allocation approach to achieve the optimal solution to the UAV communication networks energy optimization issue. The theoretic analysis and simulation results show that the UAV communication network's forwarding allocation would be stable and energy cost would be optimal after the proposed intelligent learning course.

\section{Conflicts of Interest}

The authors declare that they have no conflicts of interest.

\section{Acknowledgments}

The work is supported by the National Natural Science Foundation of China (nos. 61702543, 61271254, 71501186, and 71401176).

\section{References}

[1] S. Hayat, E. Yanmaz, and R. Muzaffar, "Survey on Unmanned Aerial Vehicle Networks for Civil Applications: A Communications Viewpoint," IEEE Communications Surveys \& Tutorials, vol. 18, no. 4, pp. 2624-2661, 2016.

[2] S. Hyo-Sang and P. Segui-Gasco, UAV Communication Networkss: Selection-Making Paradigms, Encyclopedia of Aerospace Engineering, 2014.

[3] S. Rosati, K. Kruzelecki, L. Traynard, and B. Rimoldi, "Speedaware routing for UAV ad-hoc networks," in Proceedings of the 2013 IEEE Globecom Workshops, GC Wkshps 2013, pp. 13671373, USA, December 2013.

[4] Y. Zhu, Q. Huang, J. Li, and D. Wu, "Design and evaluation of airborne communication networks," in Proceedings of the 7th International Conference on Ubiquitous and Future Networks, ICUFN 2015, pp. 277-282, Japan, July 2015.

[5] G. G. Ortiz, S. Lee, S. Monacos, M. Wright, and A. Biswas, "Design and development of a robust ATP subsystem for the altair UAV-to-ground lasercomm 2.5-Gbps demonstration," in Proceedings of the SPIE - The International Society of PhotoOptical Instrumentation Engineers: Free-Space Laser Communication Technologies XV, High-Power Lasers and Applications, pp. 103-114, San Jose, CA, USA, January 2003.

[6] F. Luo, C. Jiang, J. Du et al., "A distributed gateway selection algorithm for UAV networks," IEEE Transactions on Emerging Topics in Computing, vol. 3, no. 1, pp. 22-33, 2015.

[7] C. Yin, Z. Xiao, X. Cao, X. Xi, P. Yang, and D. Wu, "Enhanced routing protocol for fast flying UAV network," in Proceedings of the 2016 IEEE International Conference on Communication Systems, ICCS 2016, pp. 1-6, China, December 2016. 
[8] Y. Saleem, M. H. Rehmani, and S. Zeadally, "Integration of Cognitive Radio Technology with unmanned aerial vehicles: Issues, opportunities, and future research challenges," Journal of Network and Computer Applications, vol. 50, pp. 15-31, 2015.

[9] D. H. Choi, S. H. Kim, and D. K. Sung, "Energy-efficient maneuvering and communication of a single UAV-based relay," IEEE Transactions on Aerospace and Electronic Systems, vol. 50, no. 3, pp. 2320-2327, 2014.

[10] A. Puri, A Survey of Unmanned Aerial Vehicles (UAV) for Traffic Surveillance, Department of Computer Science and Engineering, University of South Florida, 2005.

[11] I. Bekmezci, O. K. Sahingoz, and Ş. Temel, "Flying Ad-Hoc Networks (FANETs): a survey," Ad Hoc Networks, vol. 11, no. 3, pp. 1254-1270, 2013.

[12] H. Wang, D. Huo, and B. Alidaee, "Position unmanned aerial vehicles in the mobile Ad hoc network," Journal of Intelligent \& Robotic Systems, vol. 74, no. 1-2, pp. 455-464, 2014.

[13] F. Ono, H. Ochiai, and R. Miura, "A Wireless Relay Network Based on Unmanned Aircraft System with Rate Optimization," IEEE Transactions on Wireless Communications, vol. 15, no. 11, pp. 7699-7708, 2016.

[14] Y. Wu, B. Zhang, S. Yang, X. Yi, and X. Yang, "Energy-efficient joint communication-motion planning for relay-assisted wireless robot surveillance," in Proceedings of the IEEE INFOCOM 2017 - IEEE Conference on Computer Communications, pp. 1-9, Atlanta, GA, USA, May 2017.

[15] K. Li, W. Ni, X. Wang, R. P. Liu, S. S. Kanhere, and S. Jha, "Energy-efficient cooperative relaying for unmanned aerial vehicles," IEEE Transactions on Mobile Computing, vol. 15, no. 6, pp. 1377-1386, 2016.

[16] D. Fudenberg and D. Levine, The Theory of Learning in Games, MIT Press, 1998.

[17] P. J. M. van Laarhoven and E. H. L. Aarts, Simulated Annealing: Theory and Applications, Reidel, Holland, 1987.

[18] W. Zhong, Y. Xu, and H. Tianfield, "Game-theoretic opportunistic spectrum sharing strategy selection for cognitive MIMO multiple access channels," IEEE Transactions on Signal Processing, vol. 59, no. 6, pp. 2745-2759, 2011.

[19] J. R. Marden, G. Arslan, and J. S. Shamma, "Cooperative control and potential games," IEEE Transactions on Systems, Man, and Cybernetics, Part B: Cybernetics, vol. 39, no. 6, pp. 1393-1407, 2009.

[20] H. P. Young, Individual Strategy and Social Structure, Princeton University Press, Princeton, NJ, USA, 1998. 


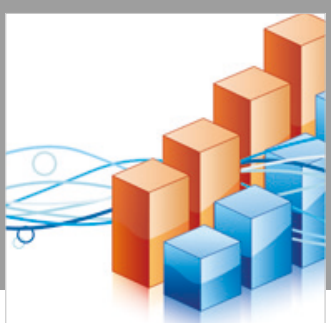

Advances in

Operations Research

\section{-n-m}
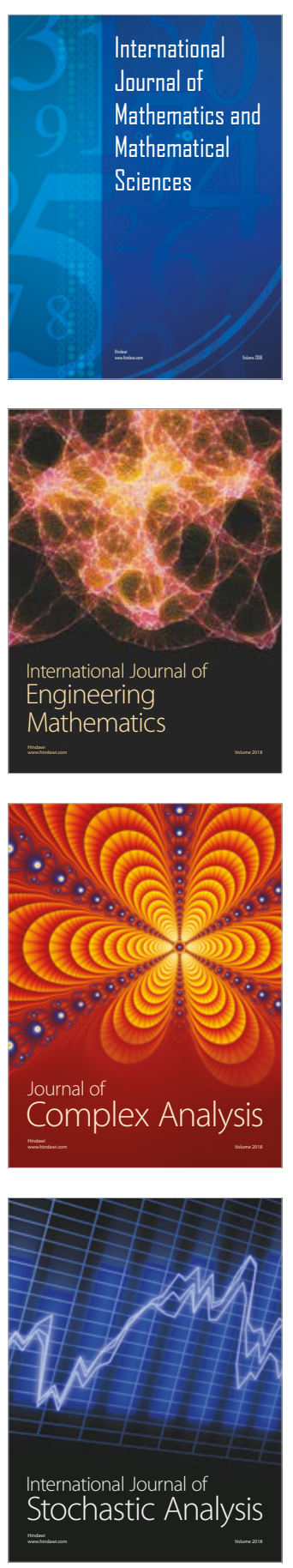
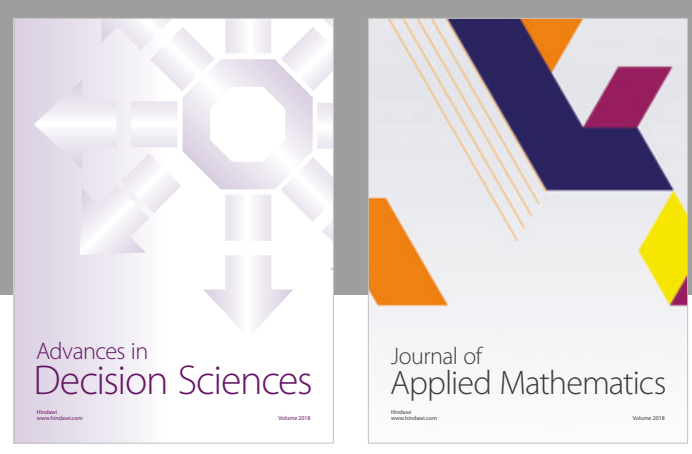

Journal of

Applied Mathematics
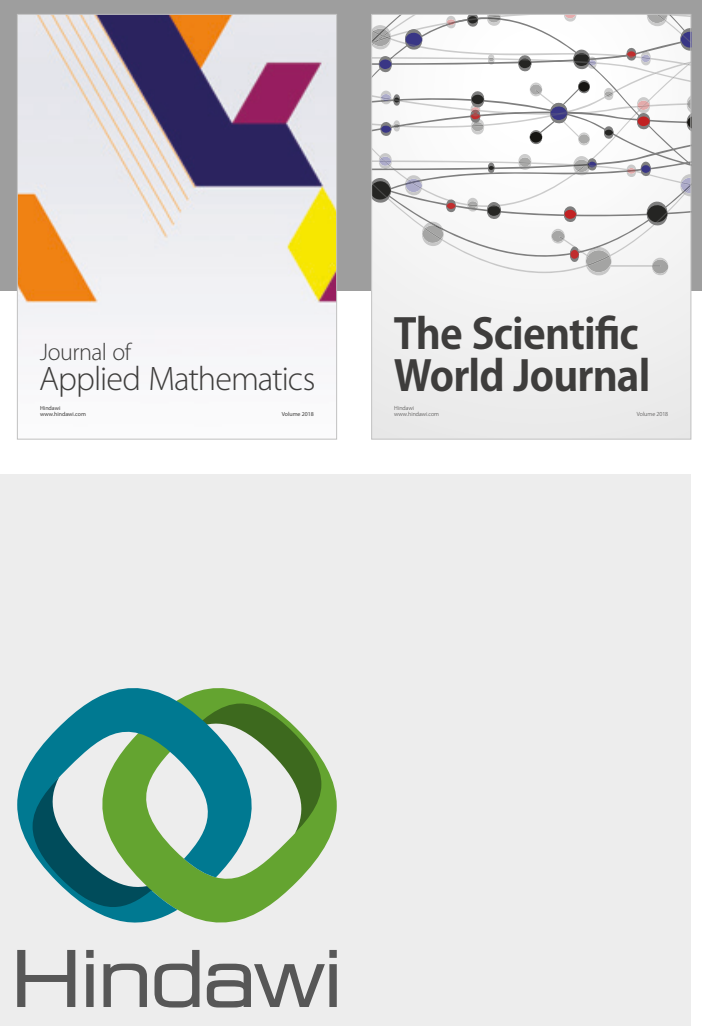

Submit your manuscripts at

www.hindawi.com

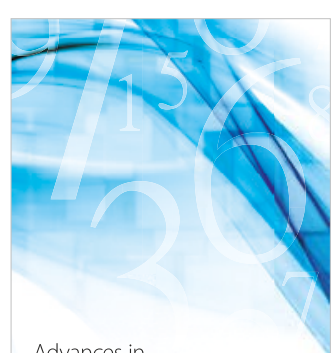

Advances in
Numerical Analysis
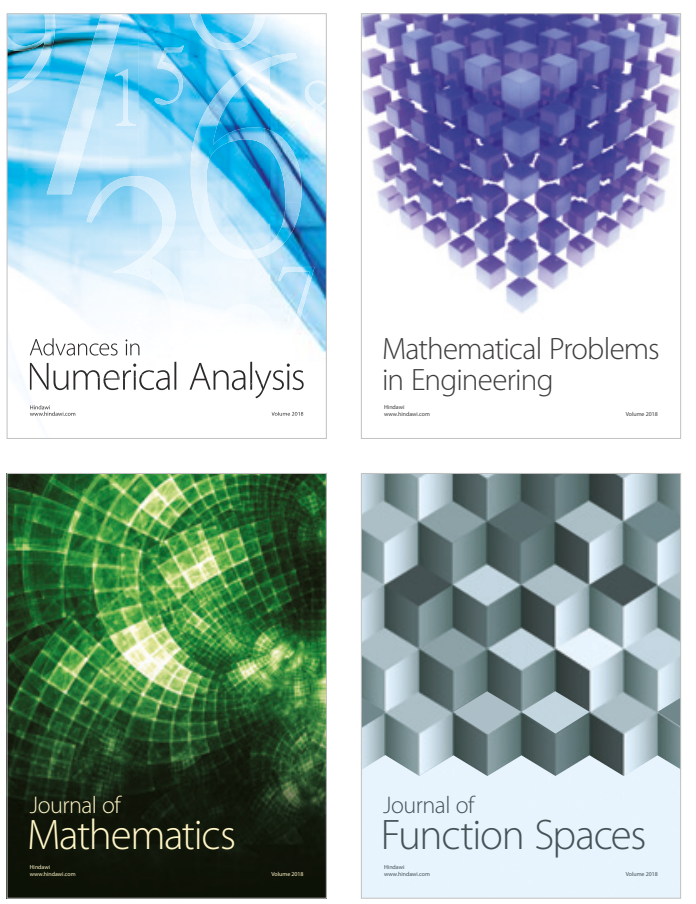

Mathematical Problems in Engineering

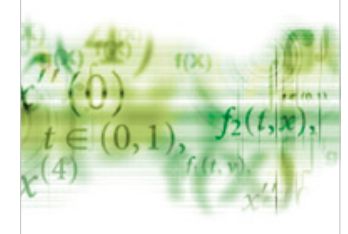

International Journal of

Differential Equations

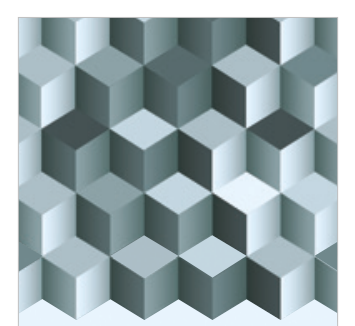

Journal of

Function Spaces
The Scientific

World Journal

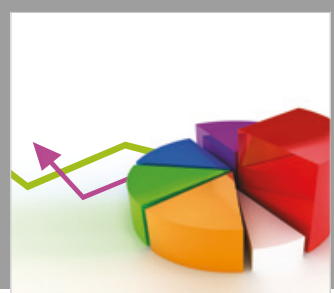

Journal of

Probability and Statistics
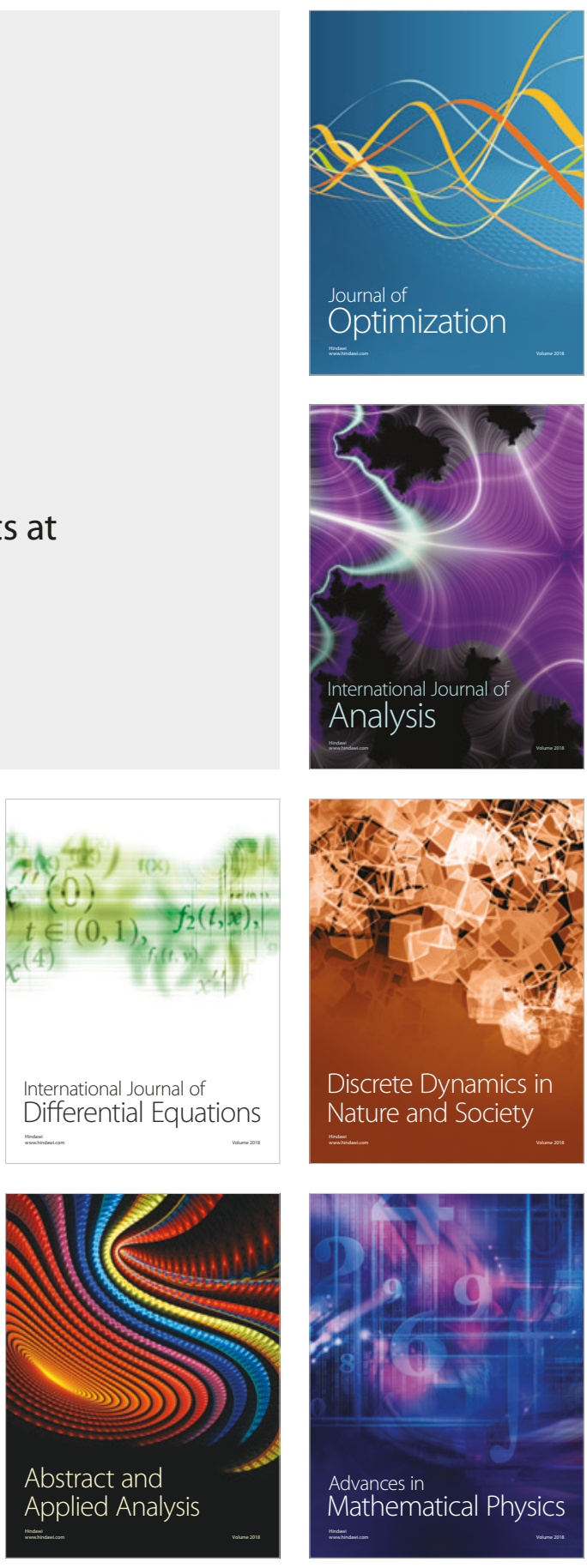